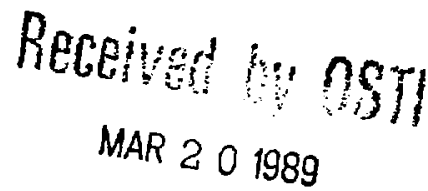

\title{
GAMMA RAYS FOR PEDESTRIANS
}

\author{
llarry .J. Lipkin \\ Argonne National Laboratory \\ Argonrle, 11, 60439 \\ and \\ Department of Nuclear Physics \\ Weizmann Institute of Science \\ Rehorot 76100 , Israel
}

\begin{abstract}
:
Nuclear gamma radiation does not have many of the properties Laken for granted in atomic or molecular radiation and necessary for lasers. The basic science and technology underlying these differences and the proposed methods of overconning difficulties resulting from them are not properly understood. Considerable illumination in this interdisciplinary problen could be provided by some back-of-the-envelope calculations and simple experimental surveys by small groups of shudents and postdoes with alt elementary knowledge of the nucleat and solid stale plysics which is evidently not Carmiliar these days to laser physicist.s.
\end{abstract}

\section{DISCLAIMER}

\begin{abstract}
This report was prepared as an account of work sponsored by an agency of the United States Government. Neither the United States Government nor any agency thereof, nor any of their employees, makes any warranty, express or implied, or assumes any legal liability or responsibility for the accuracy, completeness, or usefulness of any information, apparatus, product, or process disclosed, or represents that its use would not infringe privately owned rights. Reference herein to any specific commercial product, process, or service by trade name, trademark, manufacturer, or otherwise does not necessarily constitute or imply its endorsement, recommendation, or favoring by the United States Government or any agency thereof. The views and opinions of authors expressed herein do not necessarily state or reflect those of the United States Government or any agency thereof.
\end{abstract}

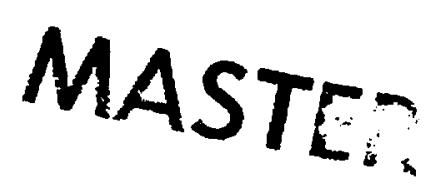

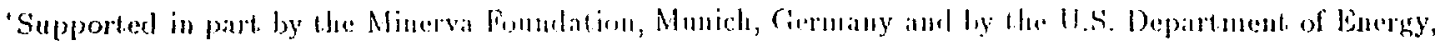

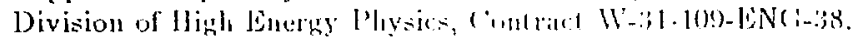


Any realistic discussien of gammat ray lascrs must take into arcommt not only the unavailablility of mirrors ame lonses used to conteol the paths of optical photoms but also the very dillerent physics presont in nuclear gamma ray transitions and not present, in the transitions at longer wave lengths used fos conventional lasers. Latser action between two energy levels depends upon the lact that the transition from the upper level to the lower level takes phace primatrily hy the emission of a photon, that the photon emithed in the transition has the right properties to cause the inverse transition in another atom or molecule, and that it is relatively asy to produce a population inversion by pumping with photons. This does not happen in nomal nuclear transitions.

The $14 \mathrm{KeV}$ anclear transition in 57 for does not normally take place by photon emission. The probablitity of photon cmission is less that $10 \%$. 'This small number of photons normally enitted do not generally lawe the proper energy to produce lhe inverse transition in another "fo mucleus. Furthermore, there is no simple way to "pump" a population inversion of these two lavels by irradiation with photons; there is only a tiny probability that a photon incident on a sample will produce a nuclear transition. The dominant absorption mechanism for photons is electronic, not nuclear, and the probability that a photon incident upon a sample will produce a nuclear transition rather than being absorbed by other means is generally less than one in a million.

These differences must be understood and taken into account before any gamma ray laser design can be considered. It is necessary to find ways to increase the probability of photon emission, to find ways to get the emitted gamma ray to have the proper energy and to find new eflicient ways of achieving a population inversion.

\section{SOME SIMPLE SCALE DIFFERENCES}

Some insight into the nature of these differences which indicate where normal intution from optical transitions breaks down for ganma rays can be scen by examining characteristic scales for the two cases. Let $R$ denote the size of the radiating system; e.g. nucleus, atorn or molecule, D denote the distance between neighboring radiators (the lattice constant in a crystal), a denote the amplitude of zero-point and thernal oscillations of the radiator, and $k$ denote the wave number of the radiation. Then for normal optical laser transitions,

$$
x \because h \sim D \because \because(1 / k)
$$


Thus

$$
k \cdot x \cdot k \sim k \cdot 1) \cdot \because 1 .
$$

for nucloar gamma ray lomolitions,

$$
R \cdot r \sim(1 / k) \cdots \cdots
$$

a.11d

$$
k R+k+k-1 \cdots k b
$$

In both cases $k h \therefore$, indicating that. lhe long wave length approximation and the multipole expansion are valid. Tut the fact that $6 x$ is of order unity for the gamma ray case and $k D$ is large introduces new problems of coherence for lasers. 'The motion of the radiating nucleus in a latice introduces a phase modulation that, tends to destroy the colnerence. The Debyc-Waller factor $e^{k-\left\langle r^{2}\right\rangle}$ expresses the renduction in intensity of the colterent radiation due to zero-point and thermal motion. The lact that $k$ is is large means that there are large phase differences between radiation from noarest neighbors, and that considerable effort is needed to control this phase in order to maintain coherence.

These effects can be seen explicilly by examining the matrix element for the anplitude emitted from two neighboring nuclei

$$
A=\left\langle\psi\left(\vec{x}_{1}, \vec{x}_{2}\right)\left|e^{i \vec{k} \cdot \dot{x}_{2}}\right| \psi\left(\vec{x}_{1}, \vec{x}_{2}\right\rangle,\right.
$$

where $\vec{x}_{1}$ and $\ddot{x}_{2}$ are the coordinates of the nuclei relative to thejr equilibrium positions. Then for gamma rays

$$
\dot{k_{\gamma}} \cdot \dot{D}>>1
$$

and

$$
k_{\gamma}^{2}\left\langle x^{2}\right\rangle \sim 1
$$

The reduction in the coherent intensity due to thermal and zero-point motion is seen to be given by the Debyc-Waller factor $e^{-k^{2}\left\langle x^{2}\right\rangle}$.

We also note that $\vec{k} \cdot \vec{D}$ satisfies 3 ragg condition for constructive interference between the two tertus in Eq. (5) only at Bragg angle. Coherence is washed out when the intensity is averaged over angles and reduces the enhancement of superradiance. 
Additional scale dillerences are simply seen in the enesgy domain; namely that the encrgy of optical radiation is much less than atomic ionization energies, while the energy of muclear gammona rays is much groalcer

$$
h w_{1, p t} \cdot H_{1} \cdot h w_{1} .
$$

This allows colergy to be lost by ionization processes absent in eonventional oplical lasers. The kinctic energy of the recoil of an atom becanse of the momentum carried by the emithed photon is much less than the nalural line width of the radiative transition in the: optical case. In the gamma ray case the recoil energy is very much targer than the natural line width and is of the same order as the thermal energy or the Debye temperature of the crystal.

$$
I \therefore \frac{\left(h k_{\gamma}\right)^{2}}{2 M} \sim k \theta_{l}
$$

For the 14 liev line in ${ }^{57} \mathrm{Fe}$

$$
\frac{\left(h k_{\gamma}\right)^{2}}{2 M}=\frac{(1.4)^{2} \times 10^{8}}{2 \times 57 \times 10^{9}} \sim \frac{1}{5 \pi 0} \text { e.v. }>1 \text {. }
$$

For a 50 keV line in a nucleus with mass $\sim 50$

$$
\frac{\left(\hbar k_{\gamma}\right)^{2}}{2 M}=\sim K T
$$

\section{SOME IMPORTANT CONSEQUENCES OF THE SCALE DIFFERENCES}

\section{A. Frequency shift effects}

1. The electromagnetic radiation emilted in a transitjon between two energy levels of an isolated atom or molecule has the right frequency to induce the inverse transition between the same two levels in another atom or molecule. The electromagnetic radiation emitted in a transition between two energy levels of an isolated nucleus does not have the right frequency to induce the inverse t,ansition between the sane two levels in another nucleus. Tho recoil kinetic energy, negligible for atomic and molexular transitions, is rencial for molene tomsitions. 
2. The ratio of the thatural line width to the energy of a muclear gannma raty can be many orders of magnitudesmaller than for the atomic or molecular casc. Thus appreciable line shilts which destroy haser action can be produced in nuclear hansitions by liny ellocts which are completely mogligible in abomir of molecular transitions. Some oxamples are Doppler shilts produced by tiny velocities, or small dillerences in external liclds acting on dillerent altoms. Nucloar transitions have cuen been aflected by the gravilational red shift of a photon passing between two nucloi.

\section{Phase Sliflt Elleets}

1. The wave length of electromagnetic radiation emitted in atomic and molecular transitions is long in comparison with the distance between matrest meighbors in normal mat.cr. "The wave length ol electromagnetic radiation emithed in nuclear transitions is short in comparison with the distance betweon nearest neighbors in normal natler. There is therefore an appreciable phase shift in the propagation of nuclear gatmma radiation between noighboring nuclei, which can aflect phatso coherence.

2. The wave length of electromagnotic radiation emitted in atomic and molecular transitions is long in comparison with the amplitude of thermal or zero point motion in normal matter. 'The wave length of electromagnetic radiation emitted in nuclear transitions is of the same order as the amplitude of thermal of zero point motion in nomal matter. 'Thus this notion can destroy phase coherence.

\section{Loss of Energy by Radiationless Transitions}

1. The dominant mechanism for the transition between an upper and a lower energy level of an isolated atom or molecule is the cmission of a photon. In most transitions of interest for grasers, the dominant mechanism lor the transition between an upper and a lower energy level of an isolated nucleus is the ejection of an atomic electron (internal conversion) not photon emission.

2. The dominant absorption mechanism in matter for photons emitted by electrons in atomic or molecular transitions is absomption by bound electrons which then make other atomic or nolecular transitions; hopefully the desired transition for a laser. The dominant absorption mechanism in maller for photons enithed by nucleons in 
mucloar transilions is still absorption hy clectrons which meoil into the continuum and camod indues other mucher transitions like the desired tramsition for a graser.

\section{NEW PROIBLEMS IN THE NUCLEAR DOMAIN RELEVANT TO GAMMA RAY LASERS}

We nom discuss the problems arising from these difforencestion The large differences in wave length. freperency, energy and monsentum between gamma radiation and optical radiation have important physical inplications both in the encrgy-momentum domain and in the space-tine domain. (iamma rays have large montenta, producing recoil shifts larger than the natural lino width, as noled in Lq. (56) They have energies larger than atomic ionization encrgics as noted in lis. (5a). Thus gamma ray entussion in nuclear transitions competes with ratiationless transitions in which the energy is carried off by an atomic electron. Tho overwhelningly dominant absorplion mechanism in matter for nuclear gamma rays is electronic, rather than muclear, and does not produce further nuclear excitation. Camma ray wave lenghts aro so much shorter than interatomic distances that phase differences botween radiation from nearest neighbors can completely destroy coherence offects as noted in ligs. (2-1). These effects all deserve serious atention. They lead to difficulties which may prove to be insurnountable.

\section{A. New Problens with the Lasing Transition}

Two important offects present in gamma ray transitions are the recoil shift and internal conversion. The recoil shift changes the energy of the gamma ray emitted in a hopefully lasing transition by such a large anount that it makes the gamma ray useless for the excitation of another nucleus. The shifted gamma ray is thus lost to further laser action. Intermal conversion takes most of the energy of the lasing transition out of the radiation ficld and converts it into incoherent electron emission also useless for further laser action. Some ways nust be found to eliminate large energy losses due to the emission of recoil-shifted gamma rays and conversion electrons in order to make the construction of a gamma ray laser feasible.

In addition the phenomenon of superradiance is very different for nuclear gamma rays, whose wave lengths are short compared to interalomic spacings, in contrast to the case of optical radiation. Most of the coherence oflect is lost in the nuclear case. 


\section{Whro Rocoil shift}

Any atom or thuclens which anits a photon must recoil with a momentum equal and opposite to the photon momentum. 'The encegy of a photon cmithed in the transition

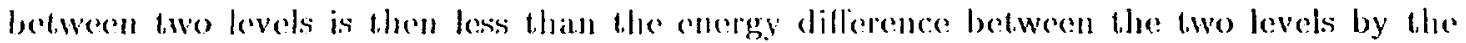
kinctic encrgy of the recoil. for optical transitions, the recoil shilt is much less than the natural line width and can be ignored. For gamman rays the recoil shift is orders of matghitude larger than the natural width as noted in Ges.(6).

The only known method for overeoming this dilliculty is by using the Mössbauer effect, in which the atom making the transition is bound in a crystal and the recoil momentum is taken up by the whole crystal with negligible energy loss. However, the probability of such a Mössbauer transition depends upon the energy of the gamma ray, the anbient temperature and the Debye temperature of the crystal in a function of these variables known as the Debye-Waller factor. The requiroment that the Debye-Waller factor must be ncar mnity to avoid wasting energy with the enision of recoil-shifted photons places serious constraints on the values of the above-mentioned varialoles.

\section{Internal Conversion}

Unlike atomic and molecular transitions, where the photon energy is smaller than ionization energies, the energy of a nuclear transition is considerably greater than the ionization energy of the atom as noted in lig. (5a). 1t is therefore possible for the transition between two nuclear levels to take place with the energy emilted by ejecting an atomic electron, rather than in the form of raliation. In common transitions, like the Mössbauer transition in ${ }^{57} \mathrm{Fe}$, the probability of this electron cjection process called internal conversion is more than an order of magnitude greater than the probability of photon emission. The only methods proposed for elininating this loss due to internal conversion are by the use of coherent effects like the Bormann elfect ${ }^{1}$ or superradiance ${ }^{2}$ which can enhance the relative probability of photon emission. Unlike the Mössbauer effect, which is well understood theoretically and confirmed experimentally, there has been no convincing theoretical prediction confirmed by experimental verification of the possibility of overcoming the loss due to internal conversion.

\section{The Bomnann Effect}

'The Bormann offect involves the propagation of colectromagnetic waves thanghgh a 
crystal in a mode which greatly reduces absorption. The crystal hatice acts somewhat like a wave guide, and there is a mode in which the fichl has a minimum or a node at the positions of the atoms in the hattice. Although this eflect reduces the absorplion of the radiation by the atoms and increases the transmission, it would generally also reduce the stimulated emission of radiation by muclei. The absence of a strong radiation field at. the nuckeus thus will not help laser operation. It has been suggested that one might be able to eal, onc's cake and have it, too, by having a. Bormann node in which the electric field vanishes at the nucleus but the magnetic fickl does not. If the internal conversion is dominated by electric multipoles and the radiative transition by magnetic nullipoles, there is a possibility of having a stimulated emission of magnetic multipole radiation while the internal conversion is suppressed. This places additional . \%nstraints on the choice of a suitable nucleas for the transition, since most nuclcar transitions are dominated by a single multipole which carries the nininum change of angular momentun, and this multipole is the sane for radiation and internal conversion. Considerable basic researcl will be needed to establish whether the Borrmann effect exists and has the desired properties in nearly perfect crystials relevant to gamina ray lasers. There will then remain the open question of how the effect is elestroyed in a practical crystal with clefects and radiation damage.

\section{Superiadiance}

Dicke las shown how the rate of radiative transitions can be enhanced by collective effects. ${ }^{2}$ There are two effects: (1) coherence in single-photon transitions involving different atoms, (2) multiphoton coherence effects.

We lirst consider the case where only a single atom in a crystal is excited, and coherent effects occur because any one of $N$ atoms may be excited and the many-body wave function is a coherent superposition of states in which a different atom is excited. The transition probability is then enhanced by a factor $N$ and the lifetime of the excited level reduced by a factor $N$ rrom the lifetime of a single atom. However, this depends upon a coherent excitation and a coherent radiation in which all contributions from different atoms are in the same phase. This is possible as long as the wave length of the radiation is large compared to the size of the radiator.

For nuclear gamma transitions this effect no longer occurs, because the interatomic distances are no longer negligible in comparison with the wave length of the radiation as shown in Eass, $(: 3-4)$. The relative plases of the contributions of radiation from different 
nuclei clepend upon the wave length of the radiation, the distance between alouss and the angle of the matiation, in a manner similar to the case of $X$-raty scattering by crystals. Although it is possible to adjust the phases of the radiation to interfere constructively in cortain directions in a crystan; eg. Bragg directions, this enhancenent occurs only in a very small solid anglo around the Bragg direction, and is mainly at the expense of radiation in other directions. The result. of the coherence is primarily to change the angular' distribution of the adiation, but not its overall intensity, thus giving lithe or no change in the lifetiune of the excited istate.

A simple derivation of this result has recontly been given." We summarize this derivation here.

Consider a system of $N$ atoms or nuclei, in which one is in an excited state and all the rest are in the ground state. The wave function for the superradiant state has an equal probability for any one of the $N$ alonns or nuclei to be in the excited state and is a coherent superposition of such singly excited systems:

$$
|S\rangle=(1 / \sqrt{N}) \sum_{i} e^{2 w_{i}}\left|g, c_{i}\right\rangle
$$

where $\left|g, e_{i}\right\rangle$ denotes the state in which the ith atom is in its excited state and all the remaining atoms are in their ground state and $\phi_{i}$ is an arbitrary phase factor chosen to maximize coherence effects. Let $J$ denote the operator describing the radiative transition to the ground state of the entire systen, denoted by $|G\rangle$, with the enission of a photon of wave vector $\vec{k}$. Then the transition matrix element is given by

$$
\{G|M| S\rangle=(1 / \sqrt{N}) \sum_{i}\left\langle C_{r}|J| g, e_{i}\right\rangle e^{i \phi_{i}} e^{-i \vec{k} \cdot \vec{r}_{i}}
$$

The intensity of the radiation is then

$$
|A|^{2}=\sum_{i} \sum_{j}|a|^{2} e^{i\left(\phi_{1}-\phi_{j}\right)} e^{-i \vec{k} \cdot\left(\vec{r}_{i}-\vec{r}_{j}\right)}
$$

where

$$
a=(1 / \sqrt{N})\left\langle G|. J| q, e_{i}\right\rangle
$$

Note that the choice

$$
\text { \% } k \cdot 1,2 n \pi
$$


gives an amplitude proportional to $N$ and an intensity proportional to $N^{2}$.

However, this coherence oceurs only in the direction of a particular wave vector k. Il wo assume that the individual sources radiatse isotropically, then the total radiation integrated over all angles is given by

$$
\int|\Lambda|^{2} d \Omega-i \pi \sum_{i} \sum_{j}|a|^{2} e^{i\left(\phi_{i}+\phi_{s}\right)} \sin \left(k\left|r_{2} \cdots r_{j}\right|\right) / k\left|r_{i} \cdots r_{j}\right| .
$$

This expression is seen to be proportional to $N$, not to $N^{2}$, if $k\left|r_{i}-r_{j}\right|>>1$. The factor $k:\left|r_{i}-r_{j}\right|$ in the denorninator immediately suppresses all contributions from pairs separated by large distances and insures than there is no $N$ dependence greater than linear for large $N$. Furbermore, the appearance of the sine rather than the cosine makes it impossible to achieve constructive interference for a set of sources equally spaced on a line. If the distance between nearest neighbors along this line is a and the overall phase diflerence between the radiation from nearest neighbors is $\phi$, then the total radiation from this line for a large crystial is

$$
\int|A|^{2} d \Omega=2 \pi N_{l}|a|^{2}\left\{1+2 \sum_{n} \sin (n \phi) / n k d\right\}
$$

where $N_{1}$ is the number of atoms in this line. The sum is clearly of order $1 / k d$, giving a contribution of order $N_{l}$ if $k d$ is of order unity. Note that if $\sin \phi=1$, then $\sin (2 \phi)=0$; $\sin (3 \phi)=-1$ and the sum will be less than $1 / k d$. If $\sin \phi$ is small and positive, the sum approaches $\pi / 2 k d$.

A dependence on $N^{2}$ is obtained only if $k\left|r_{i}-r_{j}\right|<<1$ for all pairs in the sample.

The case where many atoms are simnltaneously excited is more complicated, and the above simple argument does not hold. However, the variation of the phase from different radiators as a function of the angle must be considered. The basic pliysics can be seen by examining simple properties of multiphoton states. Consider the case where three atoms are excited and decay to the ground state by the cmission of three photons. The final state of the three photons lias the form

$$
\left.\left|\psi(123\rangle=a_{A}^{\dagger} a_{B}^{\dagger} a_{c}^{t}:\right| 0\right\rangle
$$

where $a_{A}^{\dagger}$, $a_{B}^{\dagger}$ and $a_{C}^{\dagger}$ denote the creation operators for the three photons enilled by the three atoms. If the three photons are in completely diflerent quantem states, the ware 
function $\psi$ 'Ame is normalized to mity.

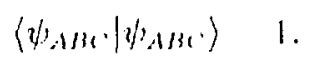

If, however, all three photons are in the same quantum state, fhe normalizalion of the stiale is $3 !-6$.

$$
\langle V A H \mid H A \lambda\rangle
$$

The transition matrix elenent for the hlore-atonn hansition will rontain a triple product of photon creation operators having the sanc form and coctlicient regardless of whether the photons are the samo or different. Thus the dillerence between the normalization factors (14a) and (14b) will appear in the final transition probability and give an culancement factor of 11 ! for the case where 11 atomis are simmltancously de-cxcited with the emission of all photons into the same quantum shate.

Let us now consider the case where the photon emitted by each atom is expressed as a linear combination of plane wave states with a phase depending upon the position of the radialing atom,

$$
a_{\mu}^{\dagger}=\frac{1}{\sqrt{S} S_{i=1}^{\dagger}} \sum_{i=1}^{s} a_{k_{i}}^{1} e^{-i \vec{k}_{i} \cdot \vec{F}_{\mu}} .
$$

where $\mu=A, B$ or $C, r_{A}, r_{B}$ and $r_{C}$ denote the positions of the three atoms, and we choose a discrete set of $S$ values of the wave number $\ddot{k}$ for convenience. For this case the norm of the state $\psi_{A B C}$ is

$$
\begin{aligned}
& \langle 0| a_{C} a_{B} a_{A} a_{A}^{\dagger}\left(a_{B}^{\dagger} a_{C}^{\dagger}|0\rangle=1+\frac{1}{S^{2}}\left\{\left|\sum_{i=1}^{S} e^{i \dot{k}_{i} \cdot\left(\dot{r}_{A}-\dot{r}_{1}\right)}\right|^{2}+\left|\sum_{i=1}^{S} e^{i \vec{k}_{i} \cdot\left(\dot{r}_{B}-\dot{r}_{i}\right)}\right|^{2}+\left|\sum_{i=1}^{S} e^{i \vec{k}_{i} \cdot\left(\dot{r}_{1} \cdot-\vec{r}_{A}\right)}\right|^{2}\right\}\right. \\
& +\frac{1}{S^{3}}\left\{\sum_{i=1}^{s} e^{i \ddot{k}_{i} \cdot\left(\dot{r}_{A}-\dot{r}_{B}\right)} \sum_{i=1}^{S} e^{i \ddot{k}_{i} \cdot\left(\ddot{r}_{B}-\vec{r}_{1}\right)} \sum_{i=1}^{S} e^{i \ddot{k}_{i} \cdot\left(\vec{r}_{1}-\vec{r}_{A}\right)}+c . c .\right\}
\end{aligned}
$$

Note that for the case where the distance between the atoms is small compared with the wave length of the radiation all the exponential factors become unity and Eq. (16) reduces to the form (14b) with the enhancement factor due to the coherence between the photons. For the case where the distance is large conpared with the wave length, the sum of the exponential factors become small and lid. (I6) reduces to the form (14a) with no enhancemsent factor due to to coherence. 
The summations in the relation (16) can be evaluated for the case of an isotropic angular distribution where it reduces to the same form as in lieg. (11),

$$
S_{S} \sum_{i=1}^{S} e^{i \ddot{k}_{i} \cdot\left(\dot{r}_{A} \ldots \dot{r}_{n}\right)} \frac{\sin \left(k\left|\vec{r}_{A}-\vec{r}_{A}\right|\right)}{k\left|\dot{r}_{A}-\dot{r}_{B}\right|}
$$

Por this case

$$
\begin{aligned}
& \left\langle 0\left|a_{C} a_{B} a_{A} a_{A}^{\dagger} a_{B}^{\prime} a_{C}^{\prime}\right| 0\right) \ldots 1+\frac{\sin ^{2}\left(k\left|\vec{r}_{A}-\vec{r}_{B}\right|\right)}{k^{2}\left|r_{A}-\ddot{r}_{B}\right|^{2}}+\frac{\sin ^{2}\left(k\left|\ddot{r}_{B}-\vec{r}_{C}\right|\right)}{k^{2}\left|\vec{r}_{B}-\ddot{r}_{C}\right|^{2}}+\frac{\sin ^{2}\left(k\left|\vec{r}_{C}-\ddot{r}_{A}\right|\right)}{k^{2}\left|\vec{r}_{C}-\vec{r}_{A}\right|} \\
& +2 \frac{\sin \left(k\left|\dot{r}_{A}-\dot{r}_{B}\right|\right) \sin \left(k\left|\vec{r}_{B}-\vec{r}_{C}\right|\right) \sin \left(k\left|\dot{r}_{C} \cdot \dot{r}_{A}\right|\right)}{k^{3}\left|\dot{r}_{A}-\vec{r}_{B}\right| \cdot\left|\vec{r}_{B}-\vec{r}_{C}\right| \cdot\left|\vec{r}_{C}-\vec{r}_{A}\right|} \text {. }
\end{aligned}
$$

The enhancement factor is seen to contain two types of terms (1) positive delinite terms depending only upon the distance between one pair of atoms; (2) terms depending upon all three interatomic distances with phases that are oscillating functions of these distances. We again see that eq. (17b) reduces to the two forms (14b) and (14a) for the limiting cases.

This result can be generalized to treat the case of $N$ atoms emilting $N$ photons by defining

$$
\begin{aligned}
\left|\psi_{N}\right\rangle & =\prod_{\mu=1}^{N} a_{\mu}^{\dagger} \\
\left|\psi_{N-1}\right\rangle & =\prod_{\mu=1}^{N-1} a_{\mu}^{\dagger} \\
\left|\psi_{N-2}^{\prime}\right\rangle & =\prod_{\substack{\mu=1 \\
\mu \neq 1}}^{N-\mathbf{i}} a_{\mu}^{\dagger} .
\end{aligned}
$$

where $a_{\mu}^{\dagger}$ is defined by Eq. (15).

We now note that

$$
\begin{aligned}
& \left\langle\psi_{N} \mid \psi_{N}\right\rangle=\left\langle\psi_{N-1} \mid \psi_{N-1}\right\rangle+\frac{1}{S^{2}} \sum_{p=1}^{N-1}\left|\sum_{i=1}^{S} e^{i \vec{k}_{i} \cdot\left(\vec{r}_{N}-\vec{r}_{p}\right)}\right|^{2}\left\langle\psi_{N-2}^{p} \mid \psi_{N-2}^{p}\right\rangle \\
& \frac{1}{S S^{2}} \sum_{\substack{i=1 \\
i=1}}^{N-1} \sum_{i=1}^{N-1} \sum_{i=1}^{S} e^{i \vec{k}_{i} \cdot\left(\vec{r}_{N}-\vec{r}_{n}\right)} \sum_{i=1}^{S} e^{i \vec{k}_{i} \cdot\left(\vec{r}_{N}-\vec{r}_{N}\right)}\left\langle\psi_{N-2}^{\prime \prime} \mid \psi_{N-2}^{\sigma}\right\rangle .
\end{aligned}
$$




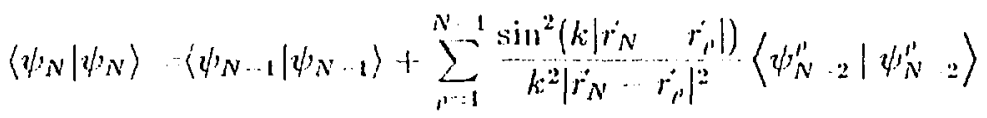

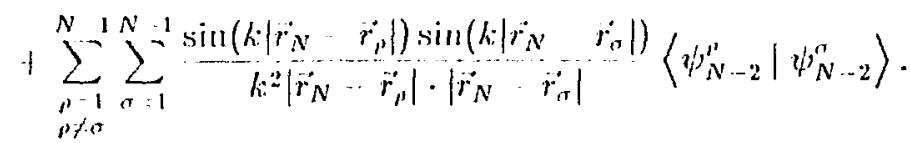

The enhancement faclor is again seen to contain two types of terms: (1) positive definite terms depending only upon the distance between one pair of aloms; (2) terms depending upon al, least three interatonic distances with phases that are oscillating functions of these distances. We again see that for the case where the distance between the atoms is small compared with the wave length of the radiation all the exponential factors become unity and Eq. (19a) reduces to the form (14b) with the enhancentent factor due to the colierence between the photons.

$$
\left\langle\psi_{N} \mid \psi_{N}\right\rangle=\left\langle\psi_{N-1} \mid \psi_{N-1}\right\rangle+(N \cdot 1)^{2}\left\langle\psi_{N-2} \mid \psi_{N-2}\right\rangle
$$

We now note that the positive definite sum is of order $N^{1 / 3}$. We can then write

$$
\left\langle\psi_{N} \mid \psi_{N}\right\rangle=\left\langle\psi_{N-1} \mid \psi_{N-1}\right\rangle+O\left(N^{1 / 3}\right)\left\langle\psi_{N-2} \mid \psi_{N-2}\right\rangle+\cdots
$$

Comparing this result with $\mathrm{Eq}$. (20a) we see that in this case there is an enhancement factor even if the distance between the atoms is large compared with the wave length of the radiation. However, it is only of order $N^{1 / 3}$ compared to the $N^{2}$ for for the case where the distance between the atoms is small compared with the wave length of the radiation.

\section{B. New Problens with the Pumping Transition}

In addition to the lasing transition itself, there is also the pumping transition necessary to achieve a high population of the excited state in the lasing transition. Here again there aie important diflerences in the nuclear case. The absorption of garnma rays by matter is very different from the absorption of optical photons, and most of the energy of the pumping transition can be expected to be wasted. Furthermore, because there will be no simple relation between the interatonic distances and the wave lengths of the pumping radiation and the lasing radiation, any collective cexcitalion of nuclei in the sample will not.

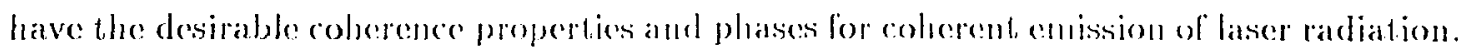




\section{Energy considerations.}

The pumping transition must have a higher energy than the lasing transition in order to reach a level higher than the lasing level, anless there is an isomeric storage level in the vicinty of the lasing level. There must then be a radiation catscade from the pumping level to the lasing level followed by the lasing transition. Because nuclear cross sections are much lower than photoelectric and Compton cross sections, the probability of absorption of pumping photons by the desired nuclear transition will be very small. Factors of $10^{-8}$ can be expected in typical cases. This juplies an enoumous waste of energy in the pumping process, and a necessity to dispose of this energy without excessively heating the sample. The Debye-Waller factor is very sensitive to temperature, and unnecessary lıating can destroy the cfliciency of the Mössbance effect. There is also the energy of the cascade radiation from the pumping level to the lasing level, which can heat the sample. In addition to heating there is also the possibility of racliation damage by all the undesired photons which can lead to crystal defects and harm colerence effects.

One might envisage an isomeric storage level very close in energy to the lasing level which would require only a small energy lor the transition to the lasing level. However, no isomers haveing this property are known and it is extremely improbable that they should exist.

\section{Phase considerations}

Since the wave length of the pumping radiation is not commensurate with that of the lasing transition, Bragg conditions for colıerent excitation and radiation of these two transitions are incompatible. Thus there is no possibility of a collective excitation of the lasing level in the sample by means of the pumping radiation to give the phase coherence necessary for constructive interference between the radiation from different nuclei in superradiant or Borrmann directions.

\section{REFERENCES}

1. G. Borrmann, Z. Phys. 42, 157 (1941).

2. R. 11. Dicke, Pliys. Rev. 93,99 (1954); C. 'P. Lee, Plyss. Rev. A 13, 1657 (1976).

3. Larry J. Lipkin, Phys. Roy. Lett. 58, 1176 (1987). 